\title{
Narrative review: fibula free flap, indications, tips, and pitfalls
}

\author{
Ashleigh M. Weyh, Rui P. Fernandes \\ Department of Oral and Maxillofacial Surgery, University of Florida College of Medicine - Jacksonville, Jacksonville, FL, USA \\ Contributions: (I) Conception and design: All authors; (II) Administrative support: All authors; (III) Provision of study materials or patients: RP \\ Fernandes; (IV) Collection and assembly of data: All authors; (V) Data analysis and interpretation: None; (VI) Manuscript writing: All authors; (VII) \\ Final approval of manuscript: All authors. \\ Correspondence to: Rui P. Fernandes, MD, DMD. Chief, Division of Head and Neck Surgery, Program Director, Department of Oral \& Maxillofacial \\ Surgery, Division of Head and Neck Surgery University of Florida - Jacksonville, 653-1 W 8th Street 2nd FL/LRC, Jacksonville, FL 32209, USA. \\ Email: rui.fernandes@jax.ufl.edu.
}

\begin{abstract}
The fibula free flap is a reliable reconstruction option, also known as the "work horse" of mandibular reconstruction. Its advantages are its size and length, ability to utilize both hard and soft tissues, and its distance from the head and neck which allows for a two team approach. Pre operatively one must evaluate perfusion to the foot, as well as vasculature supplying the flap. Many adjuncts can facilitate easier harvest, inset, and monitoring. Virtual surgical planning, SPY angiography, and the WalterLorenzTM Surgical Assist Arm are all examples. Post operatively, by utilizing enhanced recovery after surgery protocols one can standardize care, which can produce more consistent results. Utilizing step down units, instead of the intensive care unit (ICU), can also help avoid common complications such as pneumonia and delirium, as well as reduce costs. Opioid use after surgery can be reduced by utilizing multimodal pain management strategies, including use of regional anesthesia blocks and indwelling catheters. By utilizing these techniques, we have found we are able to perform fibula free flap reconstruction more efficiently, with less staff, less complications, and are able to utilize less opioids post operatively. The fibula free flap continues to be extremely important for head and neck reconstruction, due to its reliability, versatility, and ability to place dental implants for total dental rehabilitation.
\end{abstract}

Keywords: Fibula free flap; head and neck; reconstructive surgery; microvascular surgery

Received: 09 November 2020; Accepted: 16 December 2020; Published: 30 March 2021.

doi: $10.21037 /$ fomm-20-43

View this article at: http://dx.doi.org/10.21037/fomm-20-43

\section{Introduction}

The first fibular free flap (FFF) was performed by Taylor et al. in 1975 (1), however Hidalgo (2) was the first to use this flap for mandibular reconstruction, however, not until 1989. It was further popularized by Wei and colleagues (3), who were able to demonstrate the reliability of this flap consistently in a large patient series. It has since become the "work horse" of mandibular reconstruction, and is also commonly used for maxillary reconstruction. It has many advantages, namely the consistent size and length of the bone, pedicle length, and vessel diameter, as well as the ability to incorporate both hard and soft tissues (4). The bone type and thickness are also ideal for bicortical placement of dental implants, which allows for single stage resection, reconstruction, and implant placement, also known as "jaw in a day" reconstruction (5-7). The distant location of the fibula from the head and neck is also ideal, allowing for a two-team approach, so the ablative surgery and flap harvest can occur at the same time, shortening total operative time (4).

\section{Anatomy}

The fibula is a non-weight bearing bone in the lower leg. One can expect to harvest up to $25 \mathrm{~cm}$ of bone in length, with an average width dimension of $1-3 \mathrm{~cm}$. The major artery and vein are the peroneal artery, and the venae 
comitantes, with a consistent pedicle length up to $15 \mathrm{~cm}$. There are four to eight perforators to the bone at the level of the middle and distal third of the fibula, where these distal vessels are more likely septocutaneous, while the proximal perforators are usually musculocutaneous traversing through the soleus or flexor hallucis longus. Multiple osteotomies are possible due to the segmental blood supply (8).

\section{Indications and goals}

There are many causes resulting in the need for extensive mandibular or maxillary reconstruction, including both acquired defects such as malignancy, benign lesions, infection, trauma, osteoradionecrosis, as well as congenital deformities. A reconstruction of the jaw is indicated when the defect will be debilitating to the functions of swallowing, mastication, speech, and oral competence, as well as facial appearance. There is no absolute consensus on when free tissue transfer should be used over a non-vascularized bone graft, but current literature suggests that any segmental defect $\geq 6 \mathrm{~cm}$ generally will fare better with a vascularized graft, like a FFF $(9,10)$. However, the author uses a critical size defect in the range of $4-5 \mathrm{~cm}$ as a minimum cutoff for use of a FFF. This cutoff becomes even smaller in cases of anterior mandible reconstruction, or in cases where there is a history or planned radiation therapy (11). The decision to use a vascularized over a non-vascularized graft will generally favor vascularized in more complex situations such as reconstructing both bone and soft tissue, history/expected postoperative course of radiotherapy, and malignancy.

Mandibular reconstruction is complex as there are multiple goals for the final outcome, with a main focus of returning form and function as close to baseline as possible. This is first accomplished by reestablishing the continuity and normal anatomy, aiding to restore the normal function of swallowing, mastication, and speech production. Surgeons also need to strive to maintain lip competence and the size of the stoma, as well preserve tongue mobility and intelligible speech, while also achieving proper skin matching in color and texture. A large component of functional rehabilitation is the ability to place endosseous dental implants or to allow for fabrication of a conventional denture over the neomandible. In large ablative cases for cancer and cancer sequela, the goals often shift to making the patient functional and achieving socially acceptable cosmesis, as it is an impossibility to return to baseline esthetics after large tissue resection, multiple operations, and radiation. We present the following article in accordance with the Narrative Review reporting checklist (available at https://fomm.amegroups.com/article/ view/10.21037/fomm-20-43/rc).

In this review, we aim to outline fibula free flap reconstruction from the pre-operative to post-operative stages, highlighting tips and pitfalls experienced by our team.

\section{Methods}

\section{Pre-operative preparation}

Proper evaluation of the lower extremity is crucial to determine if the patient is a good candidate for fibular reconstruction, and should include evaluation for previous surgeries or trauma, history of deep vein thrombosis or peripheral vascular disease. Adequate perfusion can be assessed through clinical evaluation of the temperature of the skin, hair growth, or changes to the nail beds (12). The dominant pedicle to the fibula flap is the peroneal artery, and perfusion to the foot is provided by the remaining anterior and posterior tibial vessels, however in up to $5 \%$ of patients this is complicated by the presence of a peroneal arterial magna (13). In this situation the peroneal artery is the dominant blood supply to the foot, and the fibula cannot be used for free tissue transfer. Evaluation of blood flow in the entire leg can be evaluated ideally with computed tomography angiography to confirm the three vessels are present supplying the lower leg. The vasculature should also be assessed for blockages to blood flow, or excessive calcifications from peripheral vascular disease (4).

Proper evaluation of the head and neck site is most commonly performed with computed tomography, which also is necessary for virtual surgical planning (VSP), which can increase accuracy and efficiency in the operating room (14). VSP can be utilized in many ways, but is often used to providing surgical Stereolithic models, cutting guides for both the resection site and fibula, pre-bent or custom hardware, and guides to place the dental implants.

Prior to surgery, one must determine the laterality of the fibula free flap to be harvested, which is based on ideal geometry to connect the recipient vessels to the pedicle in a manner that does not result in tension or kinking of the blood vessels. As the lateral side of the fibula is used for fixation, and the pedicle is located at the proximal end of the bone, using left versus the right leg can be advantageous in different scenarios. In general, using the leg ipsilateral 

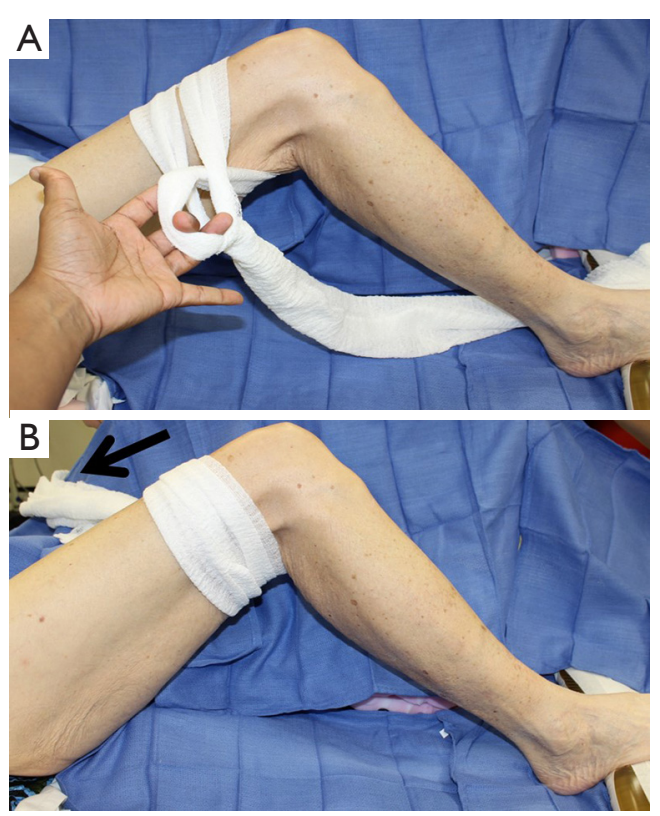

Figure $1 \mathrm{Leg}$ positioning to maximize access. A sterile rolled gauze is wrapped and looped around the leg as pictured (A), and then the free end indicated by the black arrow is secured to the opposite side of the table with an Allis clamp, or similar instrument (B).

to the defect will result in the pedicle exiting from the posterior of the flap, which is ideal for a mandibular body reconstruction using ipsilateral recipient neck vessels. If the resection involves the condyle or the ramus then the contralateral leg should be used, as the vessels will exit the fibula too posterior, and not be able to reach the recipient neck vessels without making an overly sharp turn. In a previously operated, vessel depleted neck, or for anterior reconstructions, it is also ideal to use the contralateral leg, so the pedicle will exit anterior and be in a better position for an anastomosis with the contralateral neck vessels (15).

\section{Procedure}

The procedure of raising and in setting of a fibula free flap is well documented $(4,12)$, therefore we will focus on our unique methods.

The patient is positioned supine, with the leg internally rotated so the fibula is at approximately 140 degrees to the table, with support placed for the ipsilateral hip and under the foot. The toes are prepped and covered in a manner that allows access to the dorsalis pedis to check for peripheral pulses throughout the case as needed. A sterile rolled gauze is wrapped around the leg as pictured (Figure 1), and then secured to the opposite side of the table with an Allis clamp, or similar instrument. This allows the surgeon to keep the leg stable and stationary, and positions the leg for ideal access. Instruments such as the WalterLorenzTM Surgical Assist Arm (Zimmer Biomet, Jacksonville, FL, USA) can also be utilized to improve surgical access, visualization, and efficiency, especially if the surgeon is raising the flap by oneself (Figure 2).

Use of indocyanine green laser angiography with the SPY $^{\circledR}$ Elite system (Stryker, Kalamazoo, MI, USA) is also an advantageous tool. First, the system can be used to guide in flap design by allowing surgeons to visualize perfusion in the soft tissue donor site. It can also be utilized when performing the anastomosis to judge patency of the vessels, and to determine if there is adequate perfusion to the entire free flap. Both of these uses can contribute to better overall flap success by avoiding future flap necrosis (16).

To inset and fixate the fibula, placement should be flush with the inferior border of the mandible to prevent any palpable steps. The microvascular anastomosis is carried out in normal fashion, and then the donor and recipient sites are closed. The leg often can be closed primarily if the skin paddle in $4 \mathrm{~cm}$ or less in width, or a skin graft can be used to avoid excessive tension of the closure site.

\section{Condylar resection and reconstruction}

The entire condyle should be removed if after resection there in not adequate bone stock for a plate containing at least three screw holes on the side of the condyle. If removed, a prosthetic condyle can be fixated to the fibula, or the fibula alone can be shaped to adapt to the glenoid fossa. The length of the fibula is adequate to fully reconstruct the mandible angle to angle if needed. It is either shaped and held in place by the remaining soft tissue, or it can be suspended superiorly via wire or suture outside of the fossa.

\section{Dental implants}

The height of the fibula is much shorter than the height of the native mandible, which can compromise dental rehabilitation. This can be overcome by placing the fibula just slightly above the inferior border, or by utilizing a "double barrel" technique. This involves stacking osteotomized segments on top of each other to gain height, however, this stacking can compromise the pedicle. This technique can also be difficult to use for 

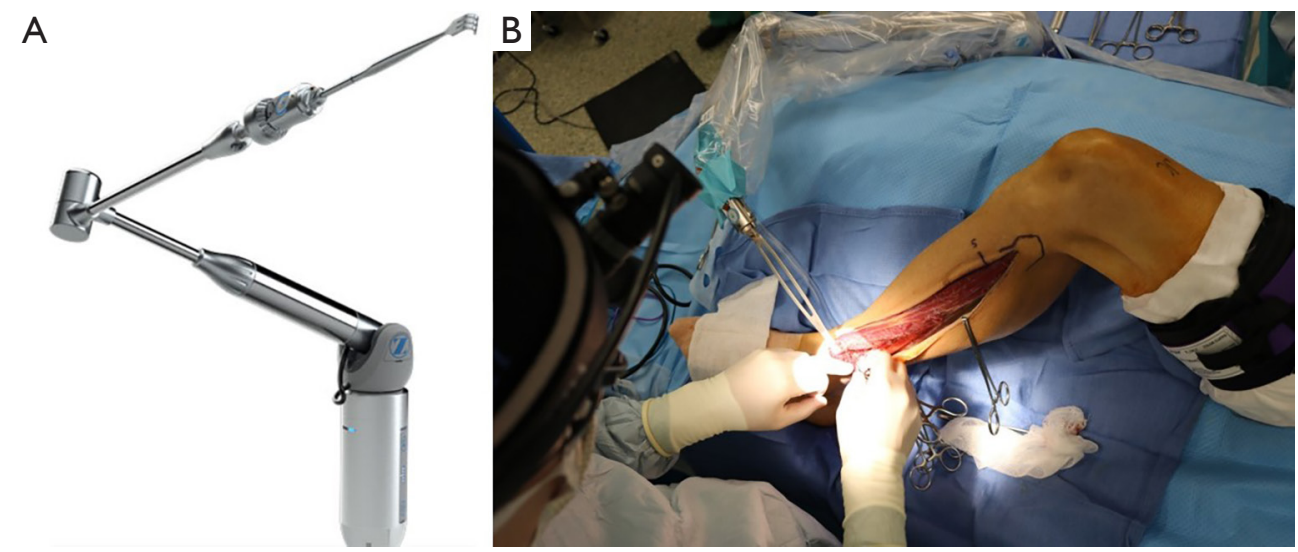

Figure 2 WalterLorenzTM Surgical Assist Arm. The WalterLorenzTM Surgical Assist Arm (A) can be utilized to improve surgical access, visualization, and efficiency, especially if the surgeon is raising the flap by oneself (B).

anterior reconstructions where multiple osteotomies are necessary (12). Due to the complexity of planning implants for the free flap, VSP should be utilized. This high complexity results from the need to plan around multiple interferences from the reconstruction plate and screws, as well as the need for precise placement and angulation of the dental implants for a future prosthesis that will need to replace multiple teeth as well as both hard and soft tissues. The implants can be planned for either immediate or delayed placement. Disadvantages of immediate implants are: final occlusion alignment may not be ideal, the implants can shift during healing stages of the fibula and the implants can add additional healing burden during this stage, and if postoperative radiation therapy will be utilized hot spots can be created around the metal implants possibly resulting in implant extrusion (8). Immediate implants are also limited to patients without major soft tissue defects, as the native oral mucosa should be present for primary closure over the implants. A case of FFF with immediate dental implant placement is demonstrated in Figure 3.

\section{Postoperative care}

Use of a standardized clinical pathway is a simple way to improve patient outcomes. Details of our protocol which has been in effect since 2015 are detailed in Yetzer et al. (17) An important aspect of our postoperative protocol is that patients are not sent to the intensive care unit (ICU), and we instead utilize a progressive/step down unit after surgery.
We have found improved outcomes by avoiding keeping patients intubated and vented in the ICU, by reducing the risk for post-operative pneumonia, infections, and delirium, as well as decreasing both patient and hospital costs. We have also recently began using an opioid sparing method of pain control via peripheral nerve blocks with indwelling catheters or wound bed catheters, to deliver continuous local anesthesia to the donor site up to three days after surgery.

Total flap loss due to vessel compromise or thrombosis is the most devastating complication, but incidence of this is less than $5 \%$ (4), which includes successfully salvaged flaps. Overall flap complication rate is $28-36 \%$, and flap takeback rate $5-25 \%$ (18). Incidence of flap compromise increases with co-morbidities, many of which are common to the oncology population, and the success of a flap salvage procedure is negatively affected by delays from the onset of ischemia time. Thus, it is important to have an established protocol for flap monitoring to avoid delays in recognition of a failing flap. Donor site morbidity is relatively minor, especially when compared to other flap harvest sites. If closed primarily it is important to monitor for compartment syndrome (12). Other noted complications specific to FFF are ankle stiffness or mild instability, motor weakness, sensory change, or a loss or reduction in the ability to run (4). As the fibula is not a weight bearing bone, patients usually can return to normal function after wearing a walking boot for approximately one month, and make a full recovery by approximately five to six weeks, with the assistance of 

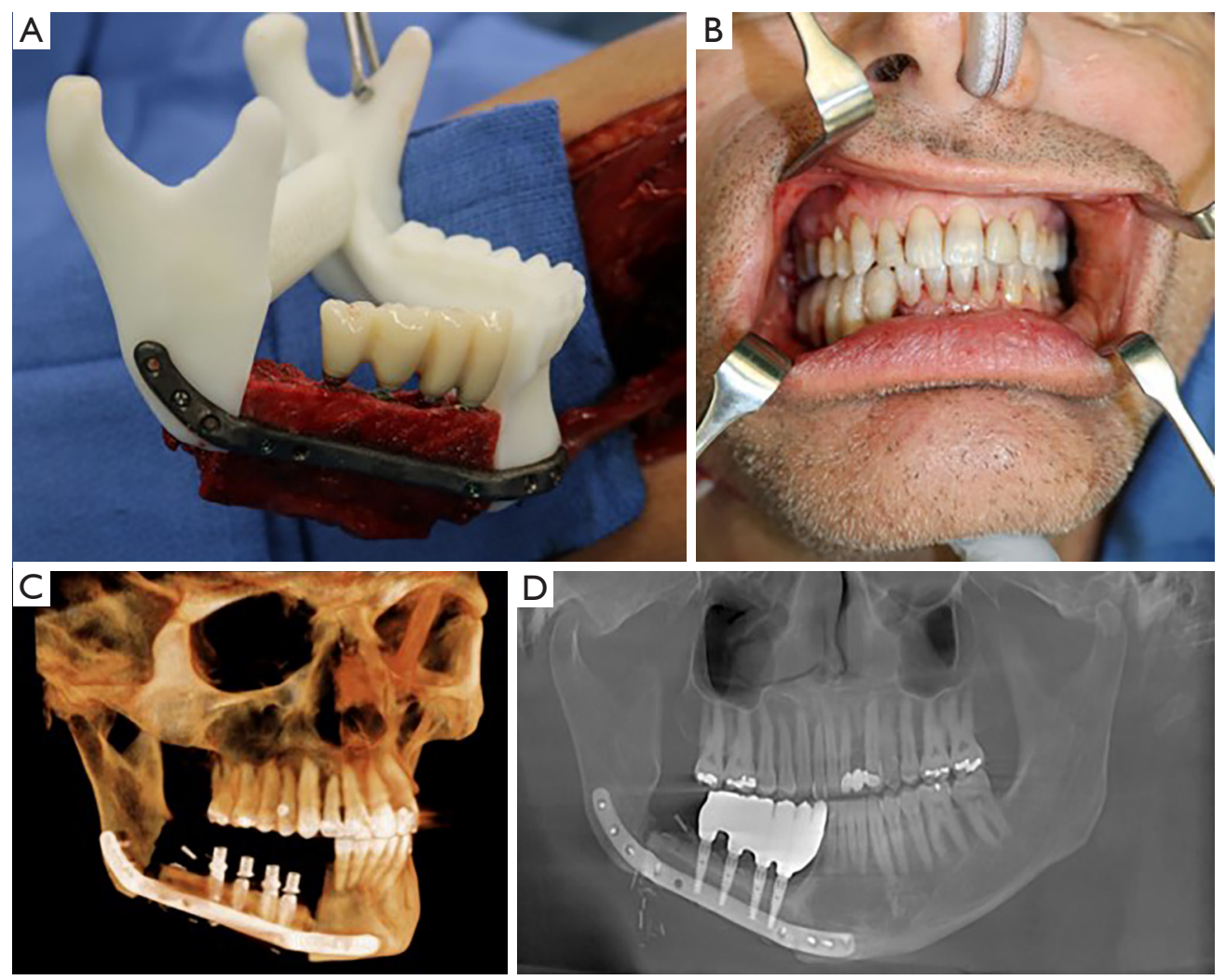

Figure 3 Case series. An example of using virtual surgical planning (VSP) to plan a first fibular free flap (FFF) reconstruction with immediate implants. (A) donor fibula segment with implants and custom reconstruction place inset into a stereolithic model. (B) intraoperative final occlusion. (C) three dimensional imaging of reconstruction. (D) postoperative panorex. Case by Dr. Rui Fernandes and Dr. Salam Salman.

physical therapy $(4,12)$.

\section{Discussion}

\section{Tips and pitfalls}

\section{Preoperative}

(I) A vascularized fibula free flap is generally preferred over a non-vascularized graft when segmental defects are $\geq 6 \mathrm{~cm}$, reconstructions requiring both bone and soft tissues, with a history of radiation, or if there is expected postoperative radiotherapy.

(II) Virtual surgical planning offers potential for increased accuracy and efficiency, while reducing time and money spent in the operating room.

(III) Determining the laterality of the donor site can be important to create ideal geometry, and prevent tension or kinking of the blood vessels, by having the pedicle project from the FFF at the ideal location.

\section{Procedure}

(I) By placing the leg in an idea position, and by utilizing adjunct instruments, such as the WalterLorenzTM Surgical Assist Arm, a surgeon can easily harvest the fibula free flap with better access, even without an assistant.

(II) Intraoperative SPY can show absolute and relative perfusion of a flap, and guide decision making if there is inadequate perfusion requiring revision of the anastomosis prior to leaving the operating room

(III) The fibula can be placed up to $1 \mathrm{~cm}$ superior to the inferior border of the mandible without causing any obvious cosmetic defects, and allowing for a more favorable implant supported prosthesis design. Alternately, the double barrel method can be used 
to increase height of the neo mandible, especially in cases where endosteal implants are planned.

\section{Postoperative}

(I) Use of regional anesthesia, specifically a popliteal indwelling catheter, can provide local anesthesia to the donor site for up to three days postoperative, helping to manage pain more effectively and with less opioids

(II) Use of a standardized free flap protocol after surgery improves outcomes and reduces costs

(III) Avoiding the ICU and prolonged mechanical ventilation after surgery can prevent pneumonia, other hospital acquired infections, and delirium, while also reducing cost.

\section{Conclusions}

The fibula free flap continues to be extremely important for head and neck reconstruction, due to its reliability, versatility, and ability to place dental implants for total dental rehabilitation. The described methods and adjuncts have all been introduced into our ever evolving standard practice, and have allowed us to perform FFF reconstructive surgery more efficiently, and with less surgical staff.

\section{Acknowledgments}

Funding: None.

\section{Footnote}

Provenance and Peer Review: This article was commissioned by the Guest Editor (Paolo Cariati) for the series "Microvascular reconstruction of head and neck oncological defects-state of the art" published in Frontiers of Oral and Maxillofacial Medicine. The article has undergone external peer review.

Reporting Checklist: The authors have completed the Narrative Review reporting checklist. Available at https://fomm. amegroups.com/article/view/10.21037/fomm-20-43/rc

Conflicts of Interest: Both authors have completed the ICMJE uniform disclosure form (available at https://fomm. amegroups.com/article/view/10.21037/fomm-20-43/coif). The series "Microvascular reconstruction of head and neck oncological defects-state of the art" was commissioned by the editorial office without any funding or sponsorship. RPF serves as an unpaid editorial board member of Frontiers of Oral and Maxillofacial Medicine from Aug 2019 to Jul 2021. He is a paid consultant for Osteomed LLC and Zimmer Biomet Holdings Inc. The authors have no other conflicts of interest to declare.

Etbical Statement: The authors are accountable for all aspects of the work in ensuring that questions related to the accuracy or integrity of any part of the work are appropriately investigated and resolved.

Open Access Statement: This is an Open Access article distributed in accordance with the Creative Commons Attribution-NonCommercial-NoDerivs 4.0 International License (CC BY-NC-ND 4.0), which permits the noncommercial replication and distribution of the article with the strict proviso that no changes or edits are made and the original work is properly cited (including links to both the formal publication through the relevant DOI and the license). See: https://creativecommons.org/licenses/by-nc-nd/4.0/.

\section{References}

1. Taylor GI, Miller GD, Ham FJ. The free vascularized bone graft. A clinical extension of microvascular techniques. Plast Reconstr Surg 1975;55:533-44.

2. Hidalgo DA. Fibula free flap: a new method of mandible reconstruction. Plast Reconstr Surg 1989;84:71-9.

3. Wei FC, Chen HC, Chuang CC, et al. Fibular osteoseptocutaneous flap: anatomic study and clinical application. Plast Reconstr Surg 1986;78:191-200.

4. Fernandes R. Fibula free flap in mandibular reconstruction. Atlas Oral Maxillofac Surg Clin North Am 2006;14:143-50.

5. Salman SO, Fernandes RP, Rawal SR. Immediate Reconstruction and Dental Rehabilitation of Segmental Mandibular Defects: Description of a Novel Technique. J Oral Maxillofac Surg 2017;75:2270.e1-8.

6. Patel A, Harrison P, Cheng A, et al. Fibular Reconstruction of the Maxilla and Mandible with Immediate ImplantSupported Prosthetic Rehabilitation: Jaw in a Day. Oral Maxillofac Surg Clin North Am 2019;31:369-86.

7. Patel SY, Kim DD, Ghali GE. Maxillofacial Reconstruction Using Vascularized Fibula Free Flaps and Endosseous Implants. Oral Maxillofac Surg Clin North Am 2019;31:259-84.

8. Shah JP, Patel SG, Singh B. Head and Neck Surgery and Oncology. Elsevier Health Sciences, 2012. 
9. Arden RL, Rachel JD, Marks SC, et al. Volume-length impact of lateral jaw resections on complication rates. Arch Otolaryngol Head Neck Surg 1999;125:68-72.

10. Foster RD, Anthony JP, Sharma A, et al. Vascularized bone flaps versus nonvascularized bone grafts for mandibular reconstruction: an outcome analysis of primary bony union and endosseous implant success. Head Neck 1999;21:66-71.

11. Nandra B, Fattahi T, Martin T, et al. Free Bone Grafts for Mandibular Reconstruction in Patients Who Have Not Received Radiotherapy: The 6-cm Rule-Myth or Reality? Craniomaxillofac Trauma Reconstr 2017;10:117-22.

12. Ward B, Kang D. Fibula. In: Kademani D, Tiwana P. editors. Atlas of Oral and Maxillofacial Surgery. Elsevier, 2015:1197-210.

13. Gaillard J, Bourcheix L, Masquelet A. Perforators of the fibular artery and suprafascial network. Surg Radiol Anat 2018;40:927-33.

14. Pucci R, Weyh A, Smotherman C, et al. Accuracy of virtual

-20-43

Cite this article as: Weyh AM, Fernandes RP. Narrative review: fibula free flap, indications, tips, and pitfalls. Front Oral Maxillofac Med 2021;3:4.

planned surgery versus conventional free-hand surgery for reconstruction of the mandible with osteocutaneous free flaps. Int J Oral Maxillofac Surg 2020;49:1153-61.

15. Al Deek NF, Tsao C, Wei F. The Surgeon's Fist with the Thumb Up to Guide the Design and Inset of the Osteoseptocutaneous Fibula Flap in Mandibular Reconstruction. Plast Reconstr Surg 2017;140:1259-62.

16. Sood M, Glat P. Potential of the SPY intraoperative perfusion assessment system to reduce ischemic complications in immediate postmastectomy breast reconstruction. Ann Surg Innov Res 2013;7:9.

17. Yetzer JG, Pirgousis P, Li Z, et al. Clinical Pathway Implementation Improves Efficiency of Care in a Maxillofacial Head and Neck Surgery Unit. J Oral Maxillofac Surg 2017;75:190-6.

18. Spiegel JH, Polat JK. Microvascular flap reconstruction by otolaryngologists: prevalence, postoperative care, and monitoring techniques. Laryngoscope 2007;117:485-90. 